\title{
DELIGNE-LUSZTIG THEORETIC DERIVATION FOR WEYL GROUPS OF THE NUMBER OF REFLECTION FACTORIZATIONS OF A COXETER ELEMENT
}

\author{
JEAN MICHEL
}

(Communicated by Pham Huu Tiep)

\begin{abstract}
Chapuy and Stump have given a nice generating series for the number of factorizations of a Coxeter element as a product of reflections. Their method is to evaluate case by case a character-theoretic expression. The goal of this note is to give a uniform evaluation of their character-theoretic expression in the case of Weyl groups, by using combinatorial properties of Deligne-Lusztig representations.
\end{abstract}

Let $W \subset \mathrm{GL}\left(\mathbb{C}^{n}\right)$ be an irreducible well-generated finite complex reflection group, let $\mathcal{R}$ be the set of its reflections, $\mathcal{R}^{*}$ the set of its reflecting hyperplanes, and let $c$ be a Coxeter element of $W$ (see [CT, Remark 1.3]).

In [CT, Theorem 1.1], Chapuy and Stump obtain a very nice generating series for the number $N_{l}:=\left|\left\{r_{1}, \ldots, r_{l} \in \mathcal{R}^{l} \mid r_{1} \ldots r_{l}=c\right\}\right|$ of factorizations of $c$ into the product of $l$ elements of $\mathcal{R}$. Their formula is

$$
\sum_{l \geq 0} \frac{t^{l}}{l !} N_{l}=\frac{1}{|W|}\left(e^{t|\mathcal{R}| / n}-e^{-t\left|\mathcal{R}^{*}\right| / n}\right)^{n} .
$$

Their method is to obtain a character-theoretic expression for $N_{l}$, that they proceed to evaluate case by case. My observation is that, in the case of Weyl groups, a uniform evaluation of their character-theoretic expression can be done using properties of Deligne-Lusztig representations 1

We start with [CT, formula above (10)], which states that if $S$ is the element of the group algebra $\mathbb{C} W$ given by $\sum_{r \in \mathcal{R}} r$, then $|W| N_{l}=\sum_{\chi \in \operatorname{Irr}(W)} \chi(1) \chi\left(S^{l} c^{-1}\right)$. We observe that $S$ is in the center of $\mathbb{C} W$, thus the formula can also be written

$$
|W| N_{l}=\sum_{\chi \in \operatorname{Irr}(W)} \chi\left(S^{l}\right) \chi\left(c^{-1}\right) .
$$

For $\chi \in \operatorname{Irr}(W)$, write the fake degree of $\chi$ as $x^{e_{1}}+\ldots+x^{e_{\chi(1)}}$ and define $N\left(\chi^{*}\right)=$ $\sum_{i=1}^{\chi(1)} e_{i}$, where $\chi^{*}$ denotes the complex conjugate (see for example [BM, $\S 4$.B]). We will need the following property:

Lemma 1. Assume that $\left|C_{W}(H)\right|$ has a common value e for all $H \in \mathcal{R}^{*}$. Then $\chi(S)=|\mathcal{R}| \chi(1)-N(\chi)-N\left(\chi^{*}\right)$.

\footnotetext{
Received by the editors October 13, 2014 and, in revised form, February 5, 2015.

2010 Mathematics Subject Classification. Primary 20F55, 5E10.

${ }^{1}$ The author thanks Christian Stump for making him aware of the problem and for a careful reading of this text.
} 
Proof. For $H \in \mathcal{R}^{*}$, whose fixator $C_{W}(H)$ is generated by a reflection $s_{H}$ of hyperplane $H$ and non-trivial eigenvalue $\zeta=\exp (2 i \pi / e)$, let $m_{H, i}(\chi)$ be the multiplicity of the eigenvalue $\zeta^{i}$ of $s_{H}$ in the representation affording $\chi$. Then we get

$$
\chi\left(\sum_{j=1}^{e-1} s_{H}^{j}\right)=\sum_{i=0}^{e-1} m_{H, i}(\chi) \sum_{j=1}^{e-1} \zeta^{i j}=(e-1) m_{H, 0}(\chi)-\sum_{i=1}^{e-1} m_{H, i}(\chi) .
$$

Now the formulae in $\mathrm{BM}$, Corollaire 4.2] read, in our case where $\left|C_{W}(H)\right|$ has a common value $e$,

$$
\begin{aligned}
\sum_{H \in \mathcal{R}^{*}} \sum_{i=1}^{e-1} m_{H, i}(\chi) & =\frac{N(\chi)+N\left(\chi^{*}\right)}{e} \\
\sum_{H \in \mathcal{R}^{*}} m_{H, 0}(\chi) & =\frac{|\mathcal{R}| \chi(1)}{e-1}-\frac{N(\chi)+N\left(\chi^{*}\right)}{e}
\end{aligned}
$$

whence the lemma.

Remark 2. Another expression valid without assuming $\left|C_{W}(H)\right|$ constant is $\chi(S)=$ $|\mathcal{R}| \chi(1)-N(\chi)-N(\iota(\chi))$ where $\iota$ is Opdam's involution. To see this, differentiate with respect to $x$ and then evaluate at $x=1$ formula [Ma, 6.5]; see also [Ma, 6.8].

We now restrict to the case where $W$ is the Weyl group of a connected reductive algebraic group $\mathbf{G}$ over an algebraic closure $\overline{\mathbb{F}}_{q}$ of the finite field $\mathbb{F}_{q}$ with $q$ elements. We assume that $\mathbf{G}$ is defined over $\mathbb{F}_{q}$ and denote by $F$ the Frobenius endomorphism defining the corresponding $\mathbb{F}_{q}$-structure. Let $\mathbf{T}$ be an $F$-stable maximal torus lying in an $F$-stable Borel subgroup B. We may identify $W$ with $N_{\mathbf{G}}(\mathbf{T}) / \mathbf{T}$ and we assume $\mathbf{G}$ split, which means that $F$ acts trivially on $W$. For $w \in W$, let us denote by $R_{w}$ the (virtual) character of $\mathbf{G}^{F}$ defined by the Deligne-Lusztig induction $R_{\mathbf{T}_{w}}^{\mathbf{G}}(\mathrm{Id})$, where $\mathbf{T}_{w}$ is an $F$-stable maximal torus of type $w$ (with respect to $\mathbf{T}$ ). Here $R_{w}$ is a $\overline{\mathbb{Q}}_{\ell}$-character, for some prime number $\ell$ not dividing $q$, but we will consider it as a complex character by choosing a suitable embedding $\overline{\mathbb{Q}}_{\ell} \hookrightarrow \mathbb{C}$. The set $\mathcal{E}\left(\mathbf{G}^{F}, 1\right)$ of constituents of the various $R_{w}$ is called the set of unipotent characters of $\mathbf{G}^{F}$.

The character $R_{1}$ identifies to that of $\operatorname{Ind}_{\mathrm{B}^{F}}^{\mathbf{G}^{F}} \mathrm{Id}$, and, since the commuting algebra of this representation is the Hecke algebra $\mathcal{H}$, isomorphic to the group algebra of $W$, we have a decomposition of the form $R_{1}=\sum_{\chi \in \operatorname{Irr}(W)} \chi(1) U_{\chi}$ where the $U_{\chi}$ are certain characters of $\mathbf{G}^{F}$ called the principal series unipotent characters.

The set $\mathcal{E}\left(\mathbf{G}^{F}, 1\right)$ and the values $\left\langle R_{w}, \rho\right\rangle_{\mathbf{G}^{F}}$ for $\rho \in \mathcal{E}\left(\mathbf{G}^{F}, 1\right)$ are independent of $q$; they provide an additional combinatorial structure on $W$ which can actually be entirely determined by the Hecke algebra. In the case where $W$ is the symmetric group, the characters $U_{\chi}$ exhaust the set $\mathcal{E}\left(\mathbf{G}^{F}, 1\right)$ and $\left\langle R_{w}, U_{\chi}\right\rangle_{\mathbf{G}^{F}}=\chi(w)$, so Deligne-Lusztig combinatorics bring nothing new. We could characterize their role in the current proof as enabling the use for other Weyl groups of the same features which occur in the proof in the symmetric group case.

By linearity we attach to any $a \in \mathbb{C} W$ a class function $R_{a}$ on $\mathbf{G}^{F}$, given if $a=$ $\sum_{w \in W} a_{w} w$ by $R_{a}=\sum_{w} a_{w} R_{w}$. In the particular case where $a$ is the idempotent $\frac{1}{|W|} \sum_{w \in W} \chi\left(w^{-1}\right) w$ attached to $\chi \in \operatorname{Irr}(W)$, we denote by $R_{\chi}$ the corresponding class function. Inverting, we get $R_{w}=\sum_{\chi} \chi(w) R_{\chi}$. By [Lu2, 3.19.2] we have $\left\langle R_{\chi}, R_{\psi}\right\rangle_{\mathbf{G}^{F}}=\delta_{\chi, \psi}$. It follows that for any two elements $a, b \in \mathbb{C} W$ we have 
$\left\langle R_{a}, R_{b}\right\rangle_{\mathbf{G}^{F}}=\sum_{\chi \in \operatorname{Irr}(W)} \chi(a) \overline{\chi(b)}$. Thus formula (1) becomes

$$
|W| N_{l}=\left\langle R_{S^{l}}, R_{c}\right\rangle .
$$

Lemma 3. We have $\frac{\chi(S)}{\chi(1)}=|\mathcal{R}|-a_{\chi}-A_{\chi}$, where $a_{\chi}$ (resp. $A_{\chi}$ ) is the valuation (resp. the degree) of the generic degree of $\mathcal{H}$ attached to $\chi$ (see for example [BM], $\S 2 . \mathrm{B}])$.

Proof. This is just a translation of Lemma 1 in terms of the invariants coming from the Hecke algebra. In the case of Coxeter groups in Lemma 1 we have $e=2$ and $\chi=\chi^{*}$. Thus Lemma 1 for Coxeter groups becomes $\chi(S)=\chi(1)|\mathcal{R}|-2 N(\chi)$. We then conclude by [BM, formula 4.21], which states that in Coxeter groups $N(\chi)=\chi(1) \frac{a_{\chi}+A_{\chi}}{2}$.

Lemma 4. For $\rho \in \mathcal{E}\left(\mathbf{G}^{F}, 1\right)$ and $l \in \mathbb{N}$ we have

$$
\left\langle R_{S^{l}}, \rho\right\rangle_{\mathbf{G}^{F}}= \begin{cases}\chi\left(S^{l}\right) & \text { if } \rho=U_{\chi} \text { for some } \chi \in \operatorname{Irr}(W), \\ 0 & \text { otherwise. }\end{cases}
$$

Proof. The blocks of the matrix $\left\{\left\langle R_{\chi}, \rho\right\rangle_{\mathbf{G}^{F}}\right\}_{\chi \in \operatorname{Irr}(W), \rho \in \mathcal{E}\left(\mathbf{G}^{F}, 1\right)}$ are called the Lusztig families. They constitute thus a partition $\Xi$ of $\mathcal{E}\left(\mathbf{G}^{F}, 1\right)$ such that for a "family" $\mathcal{F} \in \Xi$ we have:

- If $U_{\chi} \in \mathcal{F}, \rho \notin \mathcal{F}$, then $\left\langle R_{\chi}, \rho\right\rangle_{\mathbf{G}^{F}}=0$.

Given a family $\mathcal{F}$, the invariants $a_{\chi}$ and $A_{\chi}$ take a constant value on the $\chi \in \operatorname{Irr}(W)$ such that $U_{\chi} \in \mathcal{F}$ (see for example [Lu3, 4.23, 5.25 and 5.27]), thus $\chi(S) / \chi(1)$ takes a constant value that we will denote $c_{\mathcal{F}}$ on a family $\mathcal{F}$. Thus for $\rho \in \mathcal{F}$ we have

$$
\begin{aligned}
\left\langle R_{S^{l}}, \rho\right\rangle_{\mathbf{G}^{F}} & =\left\langle\sum_{\chi \in \operatorname{Irr}(W)} \chi\left(S^{l}\right) R_{\chi}, \rho\right\rangle_{\mathbf{G}^{F}}=\sum_{\left\{\chi \mid U_{\chi} \in \mathcal{F}\right\}} \chi\left(S^{l}\right)\left\langle R_{\chi}, \rho\right\rangle_{\mathbf{G}^{F}} \\
& =c_{\mathcal{F}}^{l} \sum_{\left\{\chi \mid U_{\chi} \in \mathcal{F}\right\}} \chi(1)\left\langle R_{\chi}, \rho\right\rangle_{\mathbf{G}^{F}}=c_{\mathcal{F}}^{l}\left\langle\sum_{\chi \in \operatorname{Irr}(W)} \chi(1) R_{\chi}, \rho\right\rangle_{\mathbf{G}^{F}} \\
& =c_{\mathcal{F}}^{l}\left\langle R_{1}, \rho\right\rangle_{\mathbf{G} F}= \begin{cases}0 & \text { unless } \rho \text { is a } U_{\chi}, \\
c_{\mathcal{F}}^{l} \chi(1)=\chi\left(S^{l}\right) & \text { if } \rho=U_{\chi} .\end{cases}
\end{aligned}
$$

Lemma 5. If $c$ is a Coxeter element and $\chi \in \operatorname{Irr}(W)$, then $\left\langle R_{c}, U_{\chi}\right\rangle_{\mathbf{G}^{F}}=0$ unless $\chi$ is an exterior power of the reflection character ref of $W$. Moreover, $\left\langle R_{c}, U_{\wedge^{i} \text { ref }}\right\rangle_{\mathbf{G}^{F}}=(-1)^{i}$.

Proof. Let $\mathbf{X}_{c}$ be the Deligne-Lusztig variety defining $R_{c}$, so that for $g \in \mathbf{G}^{F}$ we have $R_{c}(g)=\sum_{i}(-1)^{i} \operatorname{Trace}\left(g \mid H_{c}^{i}\left(\mathbf{X}_{c}, \overline{\mathbb{Q}}_{\ell}\right)\right)$. By [Lu1, 6.7 (ii)], applied with $I=\emptyset$ and $R_{0}=\mathrm{Id}$, we know that in each cohomology group $H_{c}^{i}\left(\mathbf{X}_{c}, \overline{\mathbb{Q}}_{\ell}\right)$ with $i=n, n+1, \ldots, 2 n$, there is exactly one irreducible representation $U_{\chi_{i}^{W}}$ in the principal series and that it has multiplicity 1 . The fact that $\chi_{i}^{W}=\wedge^{2 n-i}$ ref is [Lu1, remark 7.8] applied with $I=\emptyset$ and $R_{0}=\mathrm{Id}$. In this case the Hecke algebra $\mathcal{H}$ of loc. cit. is the same as our algebra $\mathcal{H}$ and the $i$-th power of the reflection representation of $\mathcal{H}$ defines precisely the character $U_{\wedge^{i} \text { ref }}$. 
Using Lemmas 4 and 5 to evaluate $\left(1^{\prime}\right)$ we get

$$
|W| N_{l}=\sum_{i=0}^{n}(-1)^{i} \wedge^{i} \operatorname{ref}\left(S^{l}\right) .
$$

Lemma 6. For any well-generated irreducible complex reflection group, formula $\left(1^{\prime \prime}\right)$ is equivalent to the Chapuy-Stump formula.

Proof. Since the representations $\wedge^{i}$ ref are irreducible by a result of Steinberg (see [Bou, $\S 2$ ex. 3]), formula $\left(1^{\prime \prime}\right)$ can be written

$$
|W| N_{l}=\sum_{i=0}^{n}(-1)^{i}\left(\wedge^{i} \operatorname{ref}(S) / \wedge^{i} \operatorname{ref}(1)\right)^{l} \wedge^{i} \operatorname{ref}(1) .
$$

Let us pick a reflecting hyperplane $H$, let $e=\left|C_{W}(H)\right|$ and $\zeta=\exp (2 i \pi / e)$ and let $s$ be a reflection with non-trivial eigenvalue $\zeta$ which generates $C_{W}(H)$. Let us compute $\sum_{j=1}^{j=e-1} \wedge^{i} \operatorname{ref}\left(s^{j}\right)$. We may choose a basis $e_{1}, \ldots, e_{n}$ of $\mathbb{C}^{n}$ such that $e_{i} \in$ $H$ for $i=1, \ldots, n-1$ and $s e_{n}=\zeta e_{n}$. A basis of $\wedge^{i} \mathbb{C}^{n}$ consists of $e_{I}=e_{a_{1}} \wedge \ldots \wedge e_{a_{i}}$ where $I=\left\{a_{1}, \ldots, a_{i}\right\}$ with $a_{1}<\ldots<a_{i}$ runs over all subsets of $\{1, \ldots, n\}$ of cardinality $i$. We have

$$
\sum_{j=1}^{e-1} s^{j}\left(e_{I}\right)= \begin{cases}(e-1) e_{I} & \text { if } n \notin I \\ -e_{I} & \text { otherwise }\end{cases}
$$

whence $\sum_{j=1}^{j=e-1} \wedge^{i} \operatorname{ref}\left(s^{j}\right)=(e-1)\left(\begin{array}{c}n \\ i\end{array}\right)-e\left(\begin{array}{c}n-1 \\ i-1\end{array}\right)$ and $\wedge^{i} \operatorname{ref}(S)=|\mathcal{R}|\left(\left(\begin{array}{c}n \\ i\end{array}\right)-\left(\begin{array}{c}n-1 \\ i-1\end{array}\right)\right)-$ $\left|\mathcal{R}^{*}\right|\left(\begin{array}{c}n-1 \\ i-1\end{array}\right)$. We finally get $\wedge^{i} \operatorname{ref}(S) / \wedge^{i} \operatorname{ref}(1)=|\mathcal{R}|\left(1-\frac{i}{n}\right)-\left|\mathcal{R}^{*}\right| \frac{i}{n}$ and

$$
|W| N_{l}=\sum_{i=0}^{n}(-1)^{i}\left(|\mathcal{R}|\left(1-\frac{i}{n}\right)-\left|\mathcal{R}^{*}\right| \frac{i}{n}\right)^{l}\left(\begin{array}{c}
n \\
i
\end{array}\right),
$$

which is exactly what one gets when expanding the Chapuy-Stump formula.

Note. We believe that Lemmas 4 and 5 hold for any Spetsial complex reflection group, with an appropriate definition of a formal set of unipotent characters (see [BMM]). We checked it by computer for the primitive irreducible Spetsial complex reflection groups using Mi].

For Spetsial groups whose reflections have order 2, the proof of Lemma 4 remains formally valid since Lemma 3 remains true for such groups, thus also the fact that $\chi(S) / \chi(1)$ is constant on families. For some other Spetsial groups, for example, $G_{6}$ and $G_{8}, \chi(S) / \chi(1)$ is not constant on families so the proof of Lemma 4 has to change.

\section{REFERENCES}

[Bou] N. Bourbaki, "Groupes et algèbres de Lie", Chap. 5, Hermann 1969.

[BM] Michel Broué and Jean Michel, Sur certains éléments réguliers des groupes de Weyl et les variétés de Deligne-Lusztig associées (French), Finite reductive groups (Luminy, 1994), Progr. Math., vol. 141, Birkhäuser Boston, Boston, MA, 1997, pp. 73-139. MR1429870 (98h:20077)

[BMM] Michel Broué, Gunter Malle, and Jean Michel, Split spetses for primitive reflection groups (English, with English and French summaries), Astérisque 359 (2014), vi+146. With an erratum to [MR1712862]. MR3221618

[CT] Guillaume Chapuy and Christian Stump, Counting factorizations of Coxeter elements into products of reflections, J. Lond. Math. Soc. (2) 90 (2014), no. 3, 919-939, DOI 10.1112/jlms/jdu059. MR3291807 
[Lu1] G. Lusztig, Coxeter orbits and eigenspaces of Frobenius, Invent. Math. 38 (1976/77), no. 2, 101-159. MR0453885 (56 \#12138)

[Lu2] George Lusztig, Representations of finite Chevalley groups, CBMS Regional Conference Series in Mathematics, vol. 39, American Mathematical Society, Providence, R.I., 1978. Expository lectures from the CBMS Regional Conference held at Madison, Wis., August 8-12, 1977. MR518617 (80f:20045)

[Lu3] George Lusztig, Characters of reductive groups over a finite field, Annals of Mathematics Studies, vol. 107, Princeton University Press, Princeton, NJ, 1984. MR742472 (86j:20038)

[Ma] Gunter Malle, On the rationality and fake degrees of characters of cyclotomic algebras, J. Math. Sci. Univ. Tokyo 6 (1999), no. 4, 647-677. MR.1742597 (2001e:20008)

[Mi] Jean Michel, The development version of the CHEVIE package of GAP3, J. Algebra 435 (2015), 308-336, DOI 10.1016/j.jalgebra.2015.03.031. MR3343221

Institut de Mathématiques de Jussieu - Paris Rive gauche, Université Denis Diderot, BÂtiment Sophie Germain, 75013, Paris, France 